\title{
NANOSUSPENSION: A NOVEL DRUG DELIVERY SYSTEM
}

\author{
Bhatt Ganesh, Raturi Ankita* \& Kothiyal Preeti
}

Shri Guru Ram Rai Institute of Technology \& Sciences Dehradun, Uttarakhand, India

\begin{abstract}
Low bioavailability is the major problem associated with poorly soluble drugs. The problem is more complex for drugs which are poorly soluble in both aqueous and nonaqueous media, as solubility is an essential factor for drug absorption, independent of the route of administration. Nanosuspensions have emerged as an attractive and promising approach to improve stability and bioavailability of poorly soluble drugs. These are very finely colloid, biphasic, dispersed, solid drug particles in an aqueous vehicle, size below $1 \mu \mathrm{m}$, without any matrix material, stabilized by surfactants and polymers. Techniques such as wet milling, high-pressure homogenization, emulsification-solvent evaporation and supercritical fluid have been used in the preparation of nanosuspension. Nanosuspension can be delivered by oral, parenteral, pulmonary and ocular routes.
\end{abstract}

Keywords: Nanosuspensions, Solubility, Bioavailability, methods of preparation, Homogenization, Drug Delivery

\section{Introduction}

The design and formulation of a dosage form require consideration of the physical, chemical, and biological characteristics of all the drug substances and pharmaceutical ingredients to be used in its preparation. An important property of a drug substance is solubility, especially aqueous system solubility ${ }^{[1]}$. The several formulation factors like solubility, stability ambient temperature and humidity, photostability, compatibility with solvent and excipient plays critical role in successful drug formulation. Among this aqueous solubility became an obstacle for the formulation of new molecular entities. ${ }^{[2]}$ More than $40 \%$ of the drugs coming from highthroughoutput screening are poorly soluble in water. Obviously poorly water-soluble drugs show many problems in formulating them in conventional dosage forms. One of the critical problems associated with poorly soluble drugs is too low bioavailability and erratic absorption. ${ }^{[3]}$ The problem is even more intense for drugs such as itraconazole and carbamazepine (belonging to class III as classified by Washington 1996), as they are poorly soluble in both aqueous and organic media, and for drugs having a $\log P$ value of $2^{[4]}$. Such drugs often have an erratic absorption profile and highly variable bio- availability because their performance is dissolutionrate limited and is affected by the fed/fasted state of the patient.

${ }^{*}$ Correspondence to author:

Ankita Raturi

Shri Guru Ram Rai institute of Technology \& Sciences

Dehradun, Uttarakhand,

Mobile no - 09756424729

E-mail: ankita.raturi89@gmail.com 
The solubility-dissolution behavior of a drug is a key factor to its oral bioavailability. An improvement in the solubility of poorly water-soluble drugs remains one of the most challenging tasks of drug development. The techniques that can generally overcome the problem of solubility are salt formation, micronization, use of surfactant, and use of prodrugs. However, all these techniques have certain limitations. Over the last ten years, nanoparticle engineering processes have been developed and reported for pharmaceutical applications ${ }^{[5,6]}$.

There are many conventional method for increasing the solubility of poorly soluble drugs, which include micronization ${ }^{[7]}$, solubilisation using cosolvents $^{[8]}$, salt form ${ }^{[9]}$, surfactant dispersion ${ }^{[10]}$ precipitation technique ${ }^{[11,12]}$, and oily solution. Other techniques are liposomes ${ }^{[13]}$, emulsion $^{[14,15]}$, microemulsion ${ }^{[16,17]}$, solid dispersion ${ }^{[18,19]}$ and inclusion complexation using cyclodextrin ${ }^{[20-22]}$ shows sensible achiever but lack in universal applicability to all drugs. These techniques are not applicable for those drugs which are not soluble in aqueous and organic solvents. Nanotechnology can be used to solve the problems associated with these conventional approaches for solubility and bioavailability enhancement.

Nanosuspension is favoured for compound that are insoluble in water (but are soluble in oil) with high log $P$ value, high melting point and high doses. Nanosuspension technology can also be used for drugs which are insoluble in both water and organic solvents. Hydrophobic drugs such as naproxen ${ }^{[23]}$, clofazomine ${ }^{[24]}$, bupravaquone ${ }^{[25]}$, nimesulide ${ }^{[26]}$, mititane ${ }^{[27]}$, amphotericin $\mathrm{B}^{[28]}$, omeprazole $\mathrm{e}^{[29]}$, nifedipine ${ }^{[30]}$ and spironolactone ${ }^{[31]}$, are formulated as nanosuspension. There are number of formulation approaches to resolve the problems of low solubility and low bioavailability include micronization, solublization using co-solvents, use of permeation enhancers,oily solutions, surfactant dispersions, salt formation and precipitation techniques. These techniques for solubility enhancement have some limitations and hence have limited utility in solubility enhancement. Hence there is need of some different and simple approach to tackle the formulation problems to improve their efficacy and to optimize the therapy with respect to pharmacoeconomics. The drug microparticles/micronized drug powder is transferred to drug nanoparticles by techniques like bottom up technology (precipitation) and top down technology or disintegration methods ${ }^{[3]}$.

\section{Nanosuspension}

Nanosuspension is defined as very finely colloid ${ }^{[32]}$, Biphasic $^{[33]}$,dispersed, solid drug particles in an aqueous vehicle, size below $1 \mu \mathrm{m}$ without any matrix material ${ }^{[34]}$, stabilized by surfactants $^{[35]}$ and polymers ${ }^{[36]}$, prepared by suitable methods for drug delivery ${ }^{[37]}$ application, through various routes of administration like oral ${ }^{[38]}$, topical, parenteral ${ }^{[39]}$, ocular ${ }^{[40]}$ and pulmonary routes. A Nanosuspension is a submicron colloidal dispersion of drug particles in which the particle size distribution of the drug particles is usually less than one micron with an average particle size ranging between 200 and $600 \mathrm{~nm}$. A nanosuspension not only solves the problem of poor solubility and bioavailability but also alters the pharmacokinetics of drug and that improves drug safety and efficacy. 
In nanosuspension technology, the drug is maintained in the required crystalline state with reduced particle size (i.e. increase in the surface area) leading to an increased dissolution rate and therefore improved bioavailability. ${ }^{[41]}$ Reduction of drug particles to nanometer range leads to an enhanced dissolution rate not only because of increased surface area but also because of saturation solubility ${ }^{[42]}$. The increase in saturation solubility and solution velocity of nanoparticle is due to increase of vapour pressure of the particles.

Nanosuspension have disclosed the problems associated with the delivery of poorly water -solu ble and poorly water and lipid soluble drugs and are unequalled because of their simplicity and rewards they confer over other strategies. Nano sized particles can increase solution velocity and saturation solubility because of the vapor pressure effect. In addition, the diffusional distance on the surface of drug nanoparticles is decreased, thus leading to an increased concentration gradient result to a much more pronounced increase in the dissolution velocity as compared to a micronized product. The stability of the particles obtained in the nanosuspension is attributed to their uniform particle size which is created by various manufacturing processes. The absence of particles with large differences in their size in nanosuspensions prevents the existence of different saturation solubilities and concentration gradients, consequently preventing the Ostwald ripening effect. Ostwald ripening is responsible for crystal growth and subsequently formation of microparticles. It is caused by a difference in dissolution pressure/saturation solubility between small and large particles. Molecules diffuse from the higher concentration area around small particles which have higher saturation solubility to an area around larger particles possessing a lower drug concentration. This leads to the formation of a supersaturated solution around the large particles and consequently to drug crystallization and growth of the large particles [41]. Nanosuspensions differ from nanoparticles ${ }^{[43]}$, which are polymeric colloidal carriers of drugs (Nanospheres and nanocapsules), and from solid- lipid nanoparticles ${ }^{[4]}(S L N)$, which are lipidic carriers of drug.In case of drugs that are insoluble in both water and in organic media instead of using lipidic systems nanosuspensions are used as a formulation approach. Nanosuspension formulation approach is most suitable for the compounds with high $\log \mathrm{P}$ value, high melting point and high dose ${ }^{[45]}$.The use of nanotechnology to formulate poorly water soluble drugs as nanosuspension offers the opportunity to address nature of the deficiency associated with this class of drugs. Nanosuspension has been reported to enhance absorption and bioavailability it may help to reduce the dose of the conventional oral dosage forms.

\section{Methods of prepration of nanosuspension}

There are two methods for preparation of nanosuspension. They are 'Bottom up technology' and 'Top down technology' ${ }^{[46-47]}$. Conventional methods of precipitation (Hydrosols) are called Bottom Up technology. Using a precipitation technique, the drug is dissolved in an organic solvent and this solution is mixed with a miscible antisolvent. In the water-solvent mixture the solubility is low and the drug precipitates. Precipitation has also been coupled with high shear processing. The Nanoedge process (is a registered trademarks of Baxter Inter- national Inc. and its subsidiaries) relies on the precipitation of friable materials for subsequent fragmentation under conditions of high shear and/or thermal energy ${ }^{[41]}$. This is accomplished by a combination 
of rapid precipitation and high-pressure homogenization. Rapid addition of a drug solution to an antisolvent leads to sudden supersaturation of the mixed solution, and generation of fine crystalline or amorphous solids. Precipitation of an amorphous material may be favoured at high supersaturation when the solubility of the amorphous state is exceeded. The basic advantage of precipitation technique is the use of simple and low cost equipments. The basic challenge of this technique is that during the precipitation procedure the growing of the drug crystals needs to be controlled by addition of surfactant to avoid formation of microparticles. The limitation of this precipitation technique is that the drug needs to be soluble in atleast one solvent and this solvent needs to be miscible with nonsolvent. Moreover precipitation technique is not applicable to drugs, which are simultaneously poorly soluble in aqueous and nonaqueous ${ }^{[48]}$.

\section{Top down Technology}

The 'Top down Technologies' are the disintegration methods and are preferred over the precipitation methods. The 'Top Down Technologies' include Media Milling (Nanocrystals), High Pressure Homogenization in water (Dissocubes), High Pressure Homogenization in nonaqueous media (Nanopure) and combination of Precipitation and High-Pressure Homogenization (Nanoedege). ${ }^{[4,50]}$

\section{High pressure Homogenization in water (Dissocubes)}

Homogenization involves the forcing of the suspension under pressure through a valve having a narrow aperture. Dissocubes was developed by Muller et al. in 1999 ${ }^{[51]}$. The instrument can be operated at pressure varying from $100-1500$ bars (2800-21300psi) and up to 2000 bars with volume capacity of $40 \mathrm{ml}$ (for laboratory scale).

Principle: In piston gap homogeniser particle size reduction is based on the cavitation principle. Particles are also reduced due to high shear forces and the collision of the particles against each other. The dispersion contained in $3 \mathrm{~cm}$ diameter cylinder; suddenly passes through a very narrow gap of $25 \mu \mathrm{m}$. According to Bernoulli's Law the flow volume of liquid in a closed system per cross section is constant. The reduction in diameter from $3 \mathrm{~cm}$ to $25 \mu \mathrm{m}$ leads to increase in dynamic pressure and decrease of static pressure below the boiling point of water at room temperature. Due to this water starts boiling at room temperature and forms gas bubbles, which implode when the suspension leaves the gap (called cavitation) and normal air pressure, are reached. The size of the drug nanocrystals that can be achieved mainly depends on factors like temperature, number of homogenization cycles, and power density of homogenizer and homogenization pressure.

\section{Advantages}

1. It does not cause the erosion of processed materials ${ }^{[52]}$.

2. Very dilute as well as highly concentrated nanosuspensions can be prepared by handling $1 \mathrm{mg} / \mathrm{ml}$ to $400 \mathrm{mg} / \mathrm{ml}$ drug quantity ${ }^{[53]}$.

3. It is applicable to the drugs that are poorly soluble in both aqueous and organic media.

4. It allows aseptic production of nanosuspensions for parenteral administration ${ }^{[54]}$. 


\section{Disadvantages}

1. Preprocessing like micronization of drug is required.

2. High cost instruments are required that increases the cost of dosage form.

\section{Media milling (Nanocrystals or Nanosystems)}

The method is first developed by liversidge et.al. In this method the nanosuspensions are produced using high shear media mills. The media mill consists of a milling chamber, a milling shaft and a recirculation chamber the milling medium is framed of glass, zirconium oxide or highly cross-linked polystyrene resin. The milling chamber is charged with the milling media, water, drug and stabilizer and the milling media or pearls are then roated at a very high shear rate. The milling process is performed under controlled temperatures. The high energy and shear forces generated as a result of the impaction of the milling media with the drug provide the energy input to break the microparticulate drug into nano-sized particles. The unimodal distribution profile and mean diameter of $<200$, require a time profile of $30-60 \mathrm{~min}$. The media milling procedure can successfully process micronized and non-micronized drug crystals. Once the formulation and process are optimized, very short batch to batch variation is observed in the quality of dispersion. A nanosuspension of Naproxen with a mean particle size of $300-600 \mathrm{~nm}$ was prepared using pearl mill technique ${ }^{[55]}$.

\section{Advantages:}

1. Media milling is applicable to the drugs that are poorly soluble in both aqueous and organic media.

2. Very dilute as well as highly concentrated nanosuspensions can be prepared by handling $1 \mathrm{mg} / \mathrm{ml}$ to $400 \mathrm{mg} / \mathrm{ml}$ drug quantity.

3. Nanosize distribution of final nanosize products.

\section{Disadvantages:}

1. Nanosuspensions contaminated with materials eroded from balls may be problematic when it is used for long therapy.

2. The media milling technique is time consuming.

3. Some fractions of particles are in the micrometer range.

4. Scale up is not easy due to mill size and weight.

\section{Homogenization in nonaqueous media (Nanopure)}

Nanopure is suspensions homogenized in water- free media or water mixture ${ }^{[56]}$. In the Dissocubes technology, the cavitation is the determining factor of the process. But, in contrast to water, oils and oily fatty acids have very low vapour pressure and a high boiling point. Hence, the drop of static pressure will not be sufficient enough to initiate cavitation. Patents covering disintegration of polymeric material by high- pressure homogenization mention that higher 
temperatures of about $80^{\circ} \mathrm{C}$ promoted disintegration, which cannot be used for thermolabile compounds. In nanopure technology, the drug suspensions in the non- aqueous media were homogenized at $0^{\circ} \mathrm{C}$ or even below the freezing point and hence are called "deep-freeze" homogenization. The results obtained were comparable to Dissocubes and hence can be used effectively for thermolabile substances at milder conditions.

\section{Nanoedge}

The principle involved in nanoedge is same that of the precipitation and homogenization technique. This technique has an advantage of getting smaller particle size and greater stability in short period of time. In this technique the precipitated suspension is further homogenized to get smaller particle size and to avoid crystal growth. Precipitation is performed in water using water miscible solvents, such as methanol, ethanol and isopropanol. It is desired to remove the solvent completely by including evaporation step to provide a solvent free modified starting material followed by high pressure homogenization.

\section{Nanojet technology}

This technique, called opposite stream or Nanojet technology, uses a chamber where a stream of suspension is divided into two or more parts, which colloid with each other at high pressure. The high shear force produced during the process results in particle size reduction. Equipment using this principle includes the M110L and M110S micro fluidizers (Microfluidics). Dearn prepared nanosuspensions of atovaquone using the microfluidization process ${ }^{[57]}$. The major disadvantage of this technique is the high number of passes through the microfluidizer and that the product obtained contains a relatively larger fraction of microparticles.

\section{Emulsion solvent diffusion method}

Apart from the use of emulsion as drug delivering vehicle they can also be used as templates to produce nanosuspension. The use of emulsions as templates is applicable for those drugs that are soluble in either volatile organic solvent or partially water-miscible solvent. Such solvents can be used as the dispersed phase of the emulsion. An organic solvent or mixture of solvents loaded with the drug is dispersed in the aqueous phase containing suitable surfactant with stirring to form an emulsion. The obtained emulsion was further homogenized by high pressure homogenization. After homogenization cycles the emulsion was diluted with water, homogenized by homogenizer to diffuse the organic solvents convert the droplets into solid particles. Since one particle is formed in each emulsion droplet, it is possible to control the particle size of the nanosuspension by controlling the size of the emulsion. Optimizing the surfactant composition increases the intake of organic phase and ultimately the drug loading in the emulsion. Originally methanol, ethanol, ethyl acetate, chloroform are used as organic solvents. However environmental hazards and human safety concerns about residual solvents have limited their use in routine manufacturing processes. Nanosuspension of ibuprofen ${ }^{[58]}$, diclofenac ${ }^{[59]}$ and acyclovir ${ }^{[60]}$ were prepared by this method.

\section{Supercritical fluid method}

Supercritical fluid technology can be used to produce nanoparticles from drug solutions. The various methods attempted are rapid expansion of supercritical solution process (RESS), supercritical anti-solvent process and precipitation with compressed anti-solvent process 
(PCA).The RESS involves expansion of the drug solution in supercritical fluid through a nozzle, which leads to loss of solvent power of the supercritical fluid resulting in precipitation of the drug as fine particles. The disadvantages of the above methods are use of hazardous solvents and use of high proportions of surfactants and stabilizers as compared with other techniques, particle nucleation overgrowth due to transient high super saturation, which may also result in the development of an amorphous form or another undesired polymorph. Advantages of nanosuspension are shown in table1.

Table: 1. Advantages of Nanosuspensions over conventional formulations

\begin{tabular}{|c|c|c|}
\hline $\begin{array}{l}\text { Route of } \\
\text { administration }\end{array}$ & $\begin{array}{l}\text { Disadvantages of conventional } \\
\text { formulation }\end{array}$ & Benefits of Nanosuspensions \\
\hline Oral & Slow onset of action/ poor absorption & $\begin{array}{l}\text { Rapid onset of action/ improved } \\
\text { solubility so improved bioavailability }\end{array}$ \\
\hline Ocular & Lacrimal wash off/low bioavailability & Higher bioavailability/ dose consistency \\
\hline Intravenous & ssolution/ nonspeci & Rapid dissolution/ tissue targeting \\
\hline Intramuscular & Low patient compliance due to pain & Reduced tissue irritation \\
\hline Inhalations & $\begin{array}{l}\text { Low bioavailability due to low } \\
\text { solubility }\end{array}$ & $\begin{array}{l}\text { Rapid dissolution/ high bioavailability/ } \\
\text { dose regulation }\end{array}$ \\
\hline
\end{tabular}

\section{Formulation concerns}

\section{Stabilizer}

Stabilizer plays an important role in the formulation of nanosuspensions. In the absence of an appropriate stabilizer, the high surface energy of nano-sized particles can induce agglomeration or aggregation of the drug crystals. The main functions of a stabilizer are to wet the drug particles thoroughly, and to Ostwald's ripening ${ }^{[61]}$ and agglomeration of nanosuspension in order to yield a physically stable formulation by providing steric or ionic barriers. The type and amount of stabilizer has a pronounced effect on the physical stability and in-vivo behaviour of nanosuspension. In some cases, a mixture of stabilizers is required to obtain a stable nanosuspension. The drug-to-stabilizer ratio in the formulation may vary from 1:20 to 20:1 and should be investigated for a specific case. Stabilizers that have been explored so far include cellulosics, poloxamers, polysorbates, lecithins and povidones ${ }^{[62]}$. Lecithin is a stabilizer of choice if one intends to develop a parenterally acceptable and autoclavable nanosuspension.

\section{Co-Surfactants}

The choice of co-surfactant is critical when using microemulsions to formulate nanosuspensions. Since co-surfactants can greatly influence phase behaviour, the effect of cosurfactant on uptake of the internal phase for selected microemulsion composition and on drug loading should be investigated. Although the literature describes the use of bile salts and dipotassiumglycerrhizinate as co-surfactants, various solubilizers, such as Transcutol, glycofurol, ethanol and isopropanol, can be safely used as co-surfactants in the formulation of microemulsions. 


\section{Organic Solvent}

Organic solvents are used in the formulation of nanosuspension if emulsions or microemulsions are used as template. The pharmaceutically acceptable less hazardous water miscible solvent, such as methanol, ethanol, chloroform, isopropanol and partially water miscible solvents ethyl acetate, ethyl formate, butyl lactate, triacetin, propylene carbonate, benzyl alcohol, are preferent in the formulation over the conventional hazardous solvents such as dichloromethane.

\section{Other Additives}

Nanosuspensions may contain additives such as buffers, salts, polyols, osmogent and cryoprotectant depending on either route of administration or the properties of drug moiety.

\section{Post-production processing}

Post-production processing of nanosuspensions becomes essential when the drug candidate is highly susceptible to hydrolytic cleavage or chemical degradation. Processing may also be required when the best possible stabilizer isnot able to stabilize the nanosuspension for a longer period of time or there are acceptability restrictions with respect to the desired route. Considering these aspects, techniques such as lyophillization or spray drying may be employed to produce a dry powder of nanosized drug particles. Rational selection has to be made in these unit operations considering the drug properties and economic aspects. Generally, spray drying is more economicaland convenient than lyophillization. The effect of post-production processing on the particle size of the nanosuspension and moisture content of dried nanosized drug should be given due consideration.

\section{Properties of nanosuspensions Physical Long-term Stability}

The high surface energy ofnanosized particle induces agglomeration of the drug crystals. The main function of the stabilizer is to wet the drug particles thoroughly to prevent Ostwald ripening and agglomeration of the nanosuspension and form a physically stable formulation by providing a steric or an ionic barrier. Typical examples of stabilizers used in nanosuspensions are cellulosics, poloxamer, polysorbates, lecithin, polyoleate and povidones. Lecithin may be preferred in developing parenteral nanosuspensions ${ }^{[63]}$.

\section{Internal structure of Nanosuspensions}

The high-energy input during disintegration process causes structural changes inside the drug particles. When the drug particles are exposed to high-pressure homogenisation particles are transformed from crystalline state to amorphous state. The change in state depends upon the hardness of drug, number of homogenisation cycles chemical nature of drug and power density applied by homogenizer. ${ }^{[48,64]}$

\section{Adhesiveness}

There is a distinct increase in adhesiveness of ultra-fine powders compared to coarse powders. This adhesiveness of small drug nanoparticles can be exploited for improved oral delivery of poorly soluble drugs. Improved bioavailability, improved dose proportionality, reduced fed / fasted variability, reduced inter- subject variability and enhanced absorbtion rate (both human 
and animal data $)^{[65]}$ are some of the important benchmarking effects of a drug formulated as nanoparticles in oral administration. These data have been acquired in vivo in animals but also

in humans as reported by the company NanoSystems. A drastically remarkable report is that of the increase in bioavailability for danazole from 5\% (as macrosuspension) to $82 \%$ (as nanosuspension).

\section{Crystalline state and morphology}

A potential change in the crystalline structure of nanosuspensions saying increasing the amorphous fraction in the particle or even creating completely amorphous particles is a characteristic of consideration. The application of high pressures during the production of nanosuspensions was found to promote the amorphous state ${ }^{[66]}$.

\section{Evaluation of nanosuspension}

\section{A) In-Vitro Evaluations}

1. Particle size and size distribution

2. Particle charge (Zeta Potential)

3. Crystalline state and morphology

4. Saturation solubility and dissolution velocity

\section{B) In-Vivo Evaluation}

\section{Particle Size and Size Distribution}

The mean particle size and the span of particle size distribution (polydispersity index, PI) are two important characteristic parameters because they affect the saturation solubility, dissolution rate, physical stability, even in-vivo behavior of nanosuspensions [36].It has been indicated by $\mathrm{Mu}$ " ller\& Peters (1998) that saturation solubility and dissolution velocity show considerable variation with the changing particle size of the drug 31 .Particle size distribution determines the physiochemical behavior of the formulation, such as saturation solubility, dissolution velocity, physical stability, etc. The particle size distribution can be determined by photon correlation spectroscopy (PCS), laser diffraction (LD) and coulter counter multisizer [33].PCS can even be used for determining the width of the particle size distribution (polydispersity index, $\mathrm{PI}$ ). The $\mathrm{PI}$ is an important parameter that governs the physical stability of nanosuspensions and should be as low as possible for the long-term stability of nanosuspensions. A PI value of $0.1-0.25$ indicates a fairly narrow size distribution whereas a PI value greater than 0.5 indicates a very broad distribution ${ }^{[67]}$. The coulter-counter gives the absolute number of particles per volume unit for the different size classes, and it is a more efficient and appropriate technique than LD for quantifying the contamination of nanosuspensions by micro particulate drugs ${ }^{[36]}$.of the capillaries is 5-6 $\mu \mathrm{m}$ and hence a higher particle size can lead to capillary blockade and embolism.

\section{Particle Charge}

The determination of the zeta potential of a nanosuspension is essential as itgives an idea about the physical stability of the nanosuspension. The zeta potential of a Nanosuspension is 
governed by both the stabilizer and the drug itself. In order to obtain a nanosuspension exhibiting good stability, for electrostatically stabilized nanosuspension a minimum zeta potential of $\pm 30 \mathrm{mV}$ is required wheaeas in case of combined steric and electrostatic stabilization it should be a minimum of $\pm 20 \mathrm{mV}^{[68]}$.

\section{Crystalline State and Morphology}

The assessment of the crystalline state and particle morphology together helps in understanding the polymorphic or morphological changes that a drug might undergo when subjected to nanosizing ${ }^{[36]}$. Nanosuspensions can undergo a change in the crystalline structure, which may be to an amorphous form or to other polymorphic forms because of high-pressure homogenization ${ }^{[33]}$.The changes in the solid state of the drug particles as well as the extent of the amorphous fraction can be determined by X-ray diffraction analysis 33 and supplemented by differential scanning calorimetry ${ }^{[69]}$. In order to get an actual idea of particle morphology, scanning electron microscopy is preferred.

\section{Saturation Solubility and Dissolution Velocity}

Nanosuspensions have an important advantage over other techniques, that it can increase the dissolution velocity as well as the saturation solubility. The saturation solubility of the drug in different physiological buffers as well as at different temperatures should be assessed using methods described in the literature. The investigation of the dissolution velocity of nanosuspensions reflects the advantages that can be achieved over conventional formulations, especially when designing the sustained-release dosage forms based on nanoparticulate drugs ${ }^{[70]}$.The assessment of saturation solubility and dissolution velocity helps in determining the in vitro behavior of the formulation ${ }^{[33]}$.

\section{Applications of nanosuspension}

\section{Intravenous Administration.}

One of the important applications of nanosuspension technology is the formulation of intravenously administered products. The parenteral route of administration provides a quick onset of action, rapid targeting and reduced dosage of the drug. It is the preferred route for drugs undergoing first-pass metabolism and those that are not absorbed in the GIT or degraded in the GIT. It has several advantages such as quick onset of action in case of emergency, reduction in dose of the drug and the ability to target the drug quickly to the desired site of action, especially in the case of severe infections macrophages and the pathogenic microorganisms residing in the macrophages ${ }^{[71]}$.

For administration by the parenteral route, the drug either has to be solubilized or have particle/globule size below $5 \mu \mathrm{m}$ to avoid the capillary blockade. The current approaches for parenteral delivery include salt formation, solubilization using co-solvents, micellar solutions ${ }^{[72]}$, complexation with cyclodextrins and recently liposomes ${ }^{[73]}$ There are limitations on the use of these approaches because of the limitations on their solubilization capacity and parenteral acceptability. In this regard, liposomes are much more tolerable and versatile in terms of 
parenteral delivery. However, they often suffer from problems such as physical instability, high manufacturing cost and difficulties in scale-up. Nanosuspensions would be able to solve the problems mentioned above. In addition, Nanosuspensions have been found to increase the efficacy of parenterally administered drugs.

\section{Oral administration}

The oral route is the preferred route for drug delivery because of its numerous well-known advantages but Poor solubility, incomplete dissolution, and insufficient efficacies are the major problem of oral drug administration. Nanosizing of drugs can lead to a dramatic increase in their oral absorption and subsequent bioavailability ${ }^{[70]}$. Due to smaller particle sizeand much larger surface to volume ratio, oral nanosuspensions are specially used to increasethe absorption rate and bioavailability of poorly soluble drugs. The oral administration of naproxen nanoparticles lead to an area under the curve (AUC) (0-24 h) of $97.5 \mathrm{mg}-\mathrm{h} / \mathrm{l}$ compared with just $44.7 \mathrm{mg}-\mathrm{h} / \mathrm{l}$ for naprosyn suspensions and32.7 $\mathrm{mg}$-h/l for anaprox tablets ${ }^{[74]}$. In case of azithromycin nanosuspensions, more than $65 \%$ drug was found to be dissolved in 5 hours as compared with $20 \%$ of micronized drugs.

\section{Pulmonary Drug Delivery}

Nanosuspensions may prove to be an ideal approach for delivering drugs that exhibit poor solubility in pulmonary secretions. Aqueous nanosuspension can be nebulized using mechanical or ultrasonic nebulizers for lung delivery. Because of their small size, it is likely that in each aerosol droplet at least one drug particle is contained, leading to a more uniform distribution of the drug in lungs ${ }^{[33]}$. The nanoparticulate nature of drug allows the rapid diffusion and dissolution of the drug at the site of action. At the same time the increased adhesiveness of the drug to mucosal surface offers a prolonged residence time for the drug at the absorption site. The ability of nanosuspensions to offer quick onset of action initially and then controlled release of the active moiety is highly beneficial and is required by most pulmonary diseases. Moreover as nanosuspension generally contains a very low fraction of microparticulate drug, they prevent unwanted depositie mouth and pharynx, leading to decreased local and systemic side-effects of the drug. The pharmacokinetic studies of fluticasone after the intratracheal administration of nanosuspension, showed deep lung deposition and fast lung absorption with solubility playing an important role in lung retention and duration of action ${ }^{[75]}$.

\section{Ophthalmic Drug Delivery}

Suspensions offer advantages of prolonged residence time in a cul-de-sac, which is desirable for most ocular diseases for effective treatment and avoidance of high tonicity created by water soluble drugs. Nanosuspension attains saturation solubility in the lachrymal fluid, representing an ideal approach for the ocular delivery of the hydrophobic drugs. The nanosized drug particles had shown a prolonged residual time in cul-de-sac, giving sustained release of drug ${ }^{[70]}$. Their actual performance depends on the intrinsic solubility of the drug in lachrymal fluids as it governs its release and ocular bioavailability. The sustained release of drug for specified time can be achieved by incorporating nanosuspension in hydrogel base, mucoadhesive base, or in ocular inserts. The sustained release in the cul-de-sac can also be achievedby loading the nanosuspension in the polymers. The polymeric nanosuspensions of flurbiprofen and ibuprofen 
have been successfully formulated using acrylate polymers such as Eudragit RS 100 and Eudragit RL $100^{[76]}$.

\section{Drug Targeting}

Nanosuspensions can also be used for targeted delivery as their surface properties and in vivo behavior can easily be altered by changing either the stabilizer or the milieu. The drug will be up taken by the mononuclear phagocytic system to allow regional-specific delivery. This can be used for targeting antimycobacterial, fungal or leishmanial drugs to the macrophages if the infectious pathogen is persisting intracellularly. Their versatility, ease of scale up and commercial product enable the development of commercial viable nanosuspensions for targeted delivery. The engineering of stealth nanosuspensions by using various surface coatings for active or passive targeting of the desired site is the future of targeted drug delivery systems ${ }^{[70]}$. Kayser formulated a nanosuspension of Aphidicolin to improve drug targeting against leishmania infected macrophages. He stated that the drug in the conventional form had an effective concentration (EC 50) of $0.16 \mathrm{mcg} / \mathrm{ml}$ whereas the nanosuspension formulation had an enhanced activity with an EC (50) of $0.003 \mathrm{mcg} / \mathrm{ml}$.

\section{Topical Formulations}

Drug nanoparticles can be incorporated into creams and water-free ointments. The nanocrystalline form leads to an increased saturation solubility of the drug in the topical dosage form, thus enhancing the diffusion of the drug into the skin $^{[39]}$.

\section{Bioavailability Enhancement.}

The poor oral bioavailability of the drug may be due to poor solubility, poor permeability or poorstability in the gastrointestinal tract (GIT). Nanosuspensions resolve the problem of poor bioavailability by solving the twin problems of poor solubility and poor permeability across the membrane. Bioavailability of poorly soluble oleanolic acid, a hepatoprotective agent, was improved using a nanosuspension formulation. The therapeutic effect was significantly enhanced, which indicated higher bioavailability. This was due to the faster dissolution $(90 \%$ in20 $\mathrm{min}$ ) of the Lyophilized nanosuspension powder when compared with the dissolution from a coarse powder (15\% in $20 \mathrm{~min}$ ).

\section{Conclusion}

Nanosuspension technology appear to be unique economical and yet commercially feasible approach to overcome the problem associated with drug such as poor bioavailability related with delivery of hydrophobic or lipophilic drug and those that are poorly soluble in aqueous as well as organic media, it also alters the pharmacokinetics of drug and thus improves drug safety and efficacy. It can be proved as a gift as the poorly water soluble drugs can be easily formulated into nanosuspension. Production techniques such as media milling and high-pressure homogenization have been successfully employed for large- scale production of nanosuspensions. This technology can be combined with traditional dosage forms such as tablets, capsules, pellets, and can be used for parenteral products. Attractive features, such as increased dissolution velocity, increased saturation solubility, improved bioadhesivity, versatility in surface modi- fication and ease of post-production processing, have widened the applications 
of nanosuspensions for various routes. It can be administerd through oral, parenteral, pulmonary, ocular and topical routes.

\section{References}

1. Seedher, N.; Bhatia, S. Solubility enhancement of Cox-2 inhibitors using various solvent systems. AAPS PharmSciTech 2003, 4 (3), 1-8.

2. Elaine Merisko Liversidge, Gary G. Liversidge, Eugene R.Cooper. Nanosizing: a formulation approach for poorly soluble compounds. Eur.J.Pharm.Sci.2003; 18:113-120.

3. Lipinski C.Poor Aqueous Solubility-An Industry Wide Problem in Drug Discovery. Am. Pharm. Rev., 2002; 5: 82).

4. Pouton, C. W. (2000) Lipid formulations for oral administration of drugs: non-emulsifying, selfemulsifying and 'self-micro- emulsifying' drug delivery systems. Eur. J. Pharm. Sci. 11: S93-S98

5. Verma, S.; Gokhale, R.; Burgessa, D. J. A comparative study of top-down and bottom-up approaches for the preparation of micro/nanosuspensions. Int. J. Pharm. 2009, 380, 216-222.

6. Jacobs, C.; Kayser, O.; Muller, R. H. Nanosuspensions as a new approach for the formulation for the poorly soluble drug tarazepide. Int. J. Pharm. 2000, 196, 161-164.

7. Varshosaz J, Talari R, Mostafavi SA, Nokhodchi A. Dissolution enhancement of gliclazide using in situ micronization by solvent change method. Powder Technology 2008; 187: 222-230.

8. Pahala S, Joan MA, Samuel HY. Solubilization of rapamycin. Int J Pharm 2001;213:2529.

9. Abu Serajuddin TM. Salt formation to improve drug solubility. Advanced Drug Delivery Reviews 2007; 59: 603-616.

10. Wong SM, Kellway IW, Murdan S. Enhancement of the dissolution rate and oral absorption of a poorly water soluble drug by formation of surfactant containing microparticles. IntJ Pharm 2006; 317; 61-68.

11. Marazban S, Judirh B, Xiaoxia C, Steve S, Robert O, Williams III, Keith PJ. Enhanced drug dissolution using evaporative precipitation into aqueous solution. Int J Pharm 2002; 243: 17-31.

12.True LR, Ian BG, James EH, Kevin LF, Clindy AC, Chritoper JT. Development and characterization of a scalable controlled precipitation process to enhance the dissolution of poorly soluble drugs. Pharm Res 2004; 21(11): 2048-57.

13. Riaz M. Stability and uses of liposomes. Pak Pharm Sci 1995; 8(2):69-79.

14. Floyd AG. Top ten considerations in the development of parenteral emulsions. Pharm Sci Tech 1999; 4: 134-143 
15. Nakano M. Places of emulsions in drug delivery. Adv Drug Deliv Rev 2000; 45:1-4.

16.Jadhav KR,Shaikh IM,Ambade KW, Kadam VJ. Applications of microemulsion based drug delivery system. Cur Dr Del 2006; 3(3):267-273.

17. Lawrence MJ, Rees GD. Microemulsion-based media as novel drug delivery systems. Adv Drug Deliv Rev 2000; 45: 89-121.

18.Leuner C, Dressman J. Improving drug solubility for oral delivery using solid dispersions. Eur J Pharm Biopharm 2000; 50(1):47-60.

19. Hemant NJ, Ravindra WT, Martha D, Vaishali PS, Mohammed J, Mohinder SB, Sailesh AV, Abu Serajuddin TM. Bioavailability enhancement of poorly Water-Soluble drug by solid dispersion in polyethylene glycol-polysorbate 80 mixture: Int J Pharm 2004; 269: 251-258.

20. Stella VJ, Rajewski RA. Cyclodextrins: their future in drug formulation and delivery. Pharm Res 1997; 14: 556-567.

21. Loftson T, Brewster M. Pharmaceutical applications of cyclodextrin. J Pharm Sci 1996; 85: 1017-1025.

22. Marcela L, Maria M. de Bertorello, Marcela L. Solubilization of napthoquinones by complexation wiyh hydroxypropyl-b-cyclodextrin. Int J Pharm 1997; 159: 13-18.

23. Liversidge GG, Conzentino P. Drug particle size reduction for decreasing gastric irritancy an d enhancing absorption of naproxen in rats. Int J Pharm 1995; 125:309-313.

24. Peters K, Leitzkie S, Diedderichs JE, Borner K, Hahn H, Muller RH, Ehlers S. Prepration of clofazamine nanosuspension for intravenous use and evaluation of its therapeutic efficacy in Mycobacterium avium infection. J Antimicrob Chem 2000;45: 77-83.

25.Jacobs C, Kayser O, M"uller RH. Production and characterization of mucoadhesive nanosuspensions for the formulation of bupravaquone. Int J Pharm 2001; 214:3-7.

26.Debuign F, Cuisenaire J, Jeunieau L, Masereel B, Nagy JB. Synthesis of nimesulid nanoparticles in the microemulsion epikuron/isopropyl myristate/water/n-butanol (or isopropanol). J Colloid Interface Sci 2001; 243:90-101.

27.Trotta M, Gallarete M, Pattarino F, Morel S. Emulsions containing partially water-miscible solvents for the preparation of drug nanosuspensions. J Control Release 2001; 76:119128.

28.Kayser O, Olbrich C, Yardley V, Kinderlen AF, Croft SL. Formulation of amphotericin $B$ as nanosuspension for oral administration. Int J Pharm 2003;254:73-75.

29.M"oschwitzer J, Achleitner G, Pomper H, Muller RH. Development of an intravenously injecta ble chemically stable aqueous omeprazole formulation using nanosuspension technology. Eur J Pharm Biopharm 2004;58 :615-619. 
30.Hecq J, Deleers M, Fanara D, Vranckx H, Amighi K. Preparation and characterisation of nanocrystals for solubility and dissolution rate enhancement of nifedipine. Int $\mathrm{J}$ Pharm 2005;299:167-177.

31. Langguth P, Hanafy A, Frenzel D, Grenier P, Nhamias A, Ohilg T, Vergnault G, Spahn-h Langgurugth $\mathrm{H}$. Nanosuspension formulation for low-soluble drugs: pharmacokinetic evaluation using spironolactone as model compound. Drug Dev Ind Pharm 2005;31: 319-329.

32.T. Lenhardt, G. Vergnault, P. Grenier, D. Scherer, and P. Langguth1, Evaluation of Nanosuspensions for Absorption Enhancement of Poorly Soluble Drugs: In Vitro Transport 2008 (\# 2008)DOI: 10.1208/s12248-008-9050-7

33. N Arunkumar, M Deecaraman and C Rani, Nanosuspension technology and its applications in drug delivery, Asian journalof pharmaceutics, Year : 2009 , Volume : 3 , Issue : 3 , Page : 168-173.

34.Nagaraju. $\mathrm{P}^{*}$, Krishnachaithanya. K, Srinivas. V.D.N and Padma. S.V.N, Nanosuspensions: A Promising Drug Delivery Systems, International Journal of Pharmaceutical Sciences and Nanotechnology, Volume 2, Issue 4 ,January - March 2010. SuryakantaNayak*, Dibyasundar Panda, JagannathSahoo, Nanosuspension:A novel drug delivery system, Journal of Pharmacy Research Vol.3.Issue 2.February 2010241 - 246

35. XiaohuiPu, Jin Sun, Mo Li and Zhonggui He, Formulation of Nanosuspensions as a New Approach for the Delivery of Poorly, Current Nanoscience, 2009, 5, 417-427, Bentham Science Publishers Ltd. Mahendra NAKARANI *, Priyal PATEL, Jayvadan PATEL, Pankaj PATEL , Rayasa S. R. MURTHY and Subhash S. VAGHANI, Cyclosporine A-Nanosuspension Formulation, Characterization and In Vivo Comparison with a Marketed Formulation, Sci Pharm. 2010; 78: 345-361 doi:10.3797/scipharm.0908-12

36. Liversidge GG, CundyKC.Particle size reduction for improvement of oral bioavailability of hydrophobic drugs: Absolute oralbioavailability of nanocrystallinedanazol in beagle dogs. Int $\mathrm{J}$ Pharm. 1995; 125(1): 91-97.doi:10.1016/0378-5173 (95)00122-Y

37. Peters K, Leitzke S, Diederichs JE, Borner K, Hahn H, Müller RH, Ehlers S. Preparation of a clofaziminenanosuspension for intravenous use and evaluation of its therapeutic efficacy in murine Mycobacterium aviuminfection.J AntimicrobChemoth.2000; 45: 7783.doi:10.1093/jac/45.1.77

38. Rosario P, Claudio B, Piera, F, Adriana M., Antonina, P, Giovanni P. Eudragit RS100 nanosuspensions for the ophthalmic controlled delivery of ibuprofen. Eur J Pharm Sci. 2002; 16: 53-61.doi:10.1016/S0928- 0987(02)00057-X

39. 10. Jacobs $\mathrm{C}$, Muller $\mathrm{RH}$. Production and characterization of a budesonide nanosuspension for pulmonary administration.Pharm Res. 2002; 19: 189-194.doi:10.1023/A:1014276917363 
40. Panayiotis P. Constantinides, Mahesh V. Chaubal and Robert Shorr, Advances in lipid nanodispersions for parenteral drug delivery and targeting, Advanced Drug Delivery Reviews 60 (2008) 757-767.

41. Kipp J, Wong JCT, Dotty MJ and Rebbech Cl. Microprecipitation Method of Preparing Submicron Suspension, U.S. PATENT; 2003.

42. Kesisoglou F, Panmai S, Wu. Nanosizing oral formulation development and biopharmaceutical evaluation. Adv Drug Deliv Rev 2007;59: 631-644.

43. SuryakantaNayak*, Dibyasundar Panda, JagannathSahoo, Nanosuspension:A novel drug delivery system, Journal of Pharmacy Research Vol.3.Issue 2.February 2010 241-246

44. Senthil Kumar. C, VedhaHari. B.N, Sharavanan.S.P, Subramanian. N, Punitha. S and SenthilKumar.V, Novel Metronidazole Nanosuspension as a Controlled Drug Delivery System for Anthelmintic Activity, Journal of Pharmacy Research Vol.3.Issue 10.October 2010

45. J. B. Dressman, G. L. Amidon, C. Reppas, and V. P. Shah.Dissolution testing as a prognostic tool for oral drug absorption:immediate release dosage forms. Pharm Res. 15(1):1122 (1998).

46.Keck CM, Muller RH. Drug nanocrystals of poorly soluble drugs produced by high pressure homogenization. Eur J Pharm Biopharm 2006;62 :3-16.

47. Rabinow B. Nanosuspensions in drug delivery. Nature 3 2004;785-793

48. R.H.Muller,B.H.L.Bohm and .J.Grau. Nanosuspensions : a formulation approach for poorly soluble and poorly bioavailable drugs. In D.Wise (Ed) Handbook of pharmaceutical controlled release technology.2000; 345-357.

49. Chowdary K.P.R. and MadhaviB.L.R.,Novel drug delivery technologies for insoluble drugs. Ind.Drugs.2005; 42(9): 557-563.

50. Cornelia M. Keck, Rainer H. Muller.Drugnanocrystals of poorly soluble drugs produced by high-pressure homogenisation. Eur. J. Pharm.Biopharm.2006; 62:

51. Móller RH, Grau MJ, Hildebrand GE. Increase of solubility of poorly soluble drugs by transfer to Dissocubes using high pressure homogenization. ProclntSymp Control RelBioactMater 1999; 26: 112-3.

52. K.P. Krause, O. Kayser, K. Mader, R. Gust, R.H. Muller. Heavy metal intamination of nanosuspensions produced by high- pressure homogenisation.Int J. Pharm.2000; 196: 169172. 53. K.P.Krause, R.H.Muller.Production and characterization of highly concentrated nanosuspensions by high pressure homogenisation. Int.J.Pharm.2001; 214:21- 24 .

54. Jan Moschwitzer,GeorgrAchleitner, HerberkPomper, Rainer H.Muller. Development of an intraveneously injectable chemically stable aqueous omeprazole formulation using nanosuspension. Eur. J. Pharm. Biopharm.2004; 58:615-619. 
55. Anchalee AA, Pradeep KG. Effect of arginine hydrochloride and hydroxypropyl cellulose as stabilizers on the physical stability of high drug loading nanosuspensions of poorly soluble compounds. Int J Pharm 2008; 351: 282-288.

57. Dearns R. Atovaquone pharmaceutical compositions. US Patent US 6018080, 2000

58. Kocbek P, Baumgartner S, Kristl J. Preparation and evaluation of nanosuspensions for enhancing the dissolution of poorly soluble drugs. Int J Pharm 2006; 312:179-186.

59. Francesco L, Chiara S, Guido E, Francesc M, Giaime M, Anna MF. Diclofenac nanosuspensions. Influence of preparation procedure and crystal form on drug dissolution behavior. Int J Pharm 2009; 373: 124-132.

60. Panchaxari D, Shashidhar K, Vinayak M, Anand G, Anandrao K. Polymeric ocular nanosuspension for controlled release of acyclovir: in vitro release and ocular distribution. Ira J Pharm Res 2009;8(2): 79-86.

61. (Rawlins 1982; Mu“ Iler\& Bo“" hm 1998)

62. (Liversidgeetal 1992)

63. Shah T, Patel D, Hirani J, Amin AF.Nanosuspensionsas a drug delivery systems- A comprehensice review. Drug Del Tech 2007;7: 42-53.

64. V.B.Patravale, AbhijitA.Date and R.M.Kulkarni. Nanosuspensions: a promising drug delivery strategy. J.Pharm.Pharcol.2004; 56: 827-840.

65. G.C. Liversidge, Drug nanocrystals for improved drug delivery, in: Int. Symp.Control. Release Bioact. Mater, Workshop on Particulate Drug Delivery Systems,Vol. 23, 1996

66. S.M.Wong,I.W.Kellaway, S.Murdan. Enhancement of the dissolution rate and oral absorption of a poorly water soluble drug bytechniques. Indian drugs. 2005; 42(3):149-154.

67. Chen, Y.; Liu, J.; Yang, X.; Zhao, X.; Xu, H. Oleanolic acid nanosuspensions: preparation, in-vitro characterization and enhanced hepatoprotective effect. J. Pharm. Pharmacol., 2005, 57, 259-264

68. Muller $\mathrm{RH}$, Jacobs $\mathrm{C}$, Kayser $\mathrm{O}$. Nanosuspensions as Particulate Drug Formulations in Therapy Rationale for Development and What We Can Expect for the Future. Ad. Drug Del.Rev., 2001;47:3-19.

69. Liversidge GC. Paper presented at the 23 rd International symposium of the Controlled Release Bioactive Materials Society. Workshop on Particulate Drug Delivery Systems; 1996.

70. Chen, Y.; Liu, J.; Yang, X.; Zhao, X.; Xu, H. Oleanolic acid nanosuspensions: preparation, in-vitro characterization and enhanced hepatoprotective effect. J. Pharm. Pharmacol., 2005, 57, 259-264.) 


\section{International Journal of Pharmaceutical and Life Sciences ISSN 2305-0330 \\ Volume 2, Issue 4: October 2013}

71. Peters K, Leitzke S, Diederichs JE, Borner K, Hahn H, Móller RH, et al . Preparation of a clofaziminenanosuspensions for intravenous use and evaluation of its therapeutic efficacy in murine mycobacterium avium infection. J AntimicrobChemother 2000; 45:77-83.

72. (Kim etal 2001)

73. (Dupont 2002).

74. Kayser O, Olbrich C, Yardley V, KidertenAp, Croft SL. Formulation of amphotericin-B as nanosuspension for oraladministration. Int J Pharm 2003;254:73-5.

75.PoChang Chiang, Jason W. Alsup, Yurong Lai, Yiding Hu, Bruce R. Heyde, David Tung. Evaluation of Aerosol Delivery of Nanosuspension for Pre-clinical Pulmonary Drug Delivery. Nanoscale Res Lett 2009;4 :254-261

76. (Bucolo et al2002; Pignatello et al 2002b). 